\title{
A REVIEW OF EARLY-TYPE CLOSE BINARY SYSTEMS*
}

\author{
K. C. LEUNG \\ Behlen Observatory, University of Nebraska, Lincoln, Nebr., U.S.A.
}

(Received 30 August, 1983)

\begin{abstract}
This is a review of close binary systems with very early spectral types (B, O, Of, and WR stars). We limit our selection to systems with Roche model photometric solution only. There are 10 contact systems known at present. Three of them (V701 Sco, BH Cen, and RZ Pyx) are located in the vicinity of the zero-age contact (ZC) according to a Spectral Type - Period diagram. Only the first two systems have absolute dimensions available. Both of these fall into the $\mathrm{ZC}$ zone in the $\log P-\log m$ diagram and the $\log m-\log R$ diagram. The system TU Mus was thought to be a ZC system is located in the evolved contact zone (EC) in the above diagrams. Both V701 Sco and BH Cen were thought to be having mass ratios about unity. With the much improved light curves of $\mathrm{BH}$ Cen and new analysis the mass ratio has been revised to 0.84 ! This result may favor Shu's model for early-type zero-age contact systems. The evolved systems might be undergone Case A mass exchange except for two systems (V729 Cyg and RY Sct) which might be from Case B. The systems V367 Cyg was classified by Plavec as a W Ser system may be a evolved contact system.

It is interesting to note that V729 Cyg (O7f + OfIa), UW CMa(O7f $+\mathrm{O})$ and $\mathrm{CQ}$ Cep $(\mathrm{WN} 7+\mathrm{O})$ are all highly evolved contact systems. The Max II of UW CMa and CQ Cep are displaced to 0.78 and 0.80 phase, respectively. The displacement for V729 Cyg is not known due to poor coverage at this phase. The most distinct feature going from Of to Wolf-Rayet stars is the increasing domination of emission lines. It is suspected that this phaseshifts reflect the increasing activity of mass-flow in the common envelope.

There are 8 semidetached systems with reliable absolute dimensions. It is believed that 6 of them are derived from Case A while the remaining 2 are from Case B mass exchange.
\end{abstract}

\section{Acknowledgement}

This work is supported by NSF INT8120404 grant.

\footnotetext{
* Paper presented at the Lembang-Bamberg IAU Colloquium No. 80, on 'Double Stars: Physical Properties and Generic Relations', held at Bandung, Indonesia, 3-7 June, 1983.
} 